\title{
A NOTE ON PERIODIC SOLUTIONS OF THE DELAY DIFFERENTIAL EQUATION $x^{\prime}(t)=-f(x(t-1))$
}

\author{
JIANSHE YU \\ (Communicated by Yingfei Yi)
}

Abstract. Consider the delay differential equation $x^{\prime}(t)=-f(x(t-1))$, where $f \in C(\mathbb{R}, \mathbb{R})$ is odd and satisfies $x f(x)>0$ for $x \neq 0$. When $\alpha=$ $\lim _{x \rightarrow 0} \frac{f(x)}{x}$ and $\beta=\lim _{x \rightarrow \infty} \frac{f(x)}{x}$ exist, there is at least one Kaplan-Yorke periodic solution with period 4 if $\min \{\alpha, \beta\}<\frac{\pi}{2}<\max \{\alpha, \beta\}$. When this condition is not satisfied, we present several sufficient conditions on the existence/nonexistence of such periodic solutions. It is worthy of mention that some results are on the existence of at least two Kaplan-Yorke periodic solutions with period 4 and in some cases we do not require the limits $\alpha$ and/or $\beta$ to exist. Hence our results not only greatly improve but also complement existing ones. Moreover, some of the theoretical results are illustrated with examples.

\section{INTRODUCTION}

Consider the delay differential equation

$$
x^{\prime}(t)=-f(x(t-1)),
$$

where

$$
f \in C(\mathbb{R}, \mathbb{R}) \text { is odd and satisfies } x f(x)>0 \text { for } x \neq 0 .
$$

There is a rich literature on the periodic solutions of (1.1) (see, for example, 7 , 10, 11] and the references therein). Most of the results are on periodic solutions of special types, especially the Kaplan-Yorke periodic solutions.

Note that, after scaling time, any Kaplan-Yorke periodic solution can be transformed to a Kaplan-Yorke periodic solution with period 4. A periodic solution $x: \mathbb{R} \rightarrow \mathbb{R}$ with period 4 of (1.1) is said to be a Kaplan-Yorke periodic solution with period 4 if $x$ satisfies

$$
x(t)>0 \text { for } t \in(0,1), x(-t)=-x(t) \text { and } x(t+2)=-x(t) \text { for } t \in \mathbb{R} .
$$

As shown by Kaplan and Yorke [9, such special solutions of (1.1) can be found by studying a related system of coupled ordinary differential equations.

Received by the editors March 21, 2011 and, in revised form, August 5, 2011.

2010 Mathematics Subject Classification. Primary 34K13, 58E50.

This project was supported by the National Natural Science Foundation of China (11031002) and the grant DPFC (20104410110001). 
Kaplan-Yorke Theorem. Suppose that (1.2) holds and that

$$
\alpha=\lim _{x \rightarrow 0} \frac{f(x)}{x} \quad \text { and } \quad \beta=\lim _{x \rightarrow \infty} \frac{f(x)}{x}
$$

exist (allowing either one to be 0 or $\infty$ ). If $\min \{\alpha, \beta\}<\frac{\pi}{2}<\max \{\alpha, \beta\}$, then (1.1) has a Kaplan-Yorke periodic solution with period 4.

The above Kaplan-Yorke Theorem was first proved by Kaplan and Yorke [9] under the additional assumption that $F(x)=\int_{0}^{x} f(z) d z \rightarrow \infty$ as $x \rightarrow \infty$. Later, Nussbaum [15] showed that this assumption is unnecessary. Since then, many results on existence and multiplicity of periodic solutions to delay differential equations have been established by using the same technique. To name a few, see [1, 2, 3, 4, 5, 6, 7, 8, 12, 13, 14, 16. However, almost all results on existence of multiple periodic solutions did not provide any information about the minimal period. This is one of the motivations of this paper. We show that 4 is the minimal period. Moreover, some of the above results on multiplicity are about periodic solutions of different periods. Another contribution of this paper is to establish results on existence of at least two Kaplan-Yorke periodic solutions with period 4. We mention that the technique employed in this note can also be used to obtain sufficient conditions on the existence of any number of Kaplan-Yorke periodic solutions with period 4 .

One drawback of the condition $\min \{\alpha, \beta\}<\frac{\pi}{2}<\max \{\alpha, \beta\}$ in the Kaplan-Yorke Theorem is that only the limiting behavior of the function $f$ at 0 and $\infty$ is involved. On the one hand, one may wonder what will happen if the limits do not exist. On the other hand, how will other local behavior of $f$ affect the existence of periodic solutions? Moreover, it is natural to consider the cases where the inequalities are not strict and even the cases where the inequalities are not satisfied. Such cases include $\max \{\alpha, \beta\}<\frac{\pi}{2}, \min \{\alpha, \beta\}>\frac{\pi}{2}$ and $\alpha=\beta=\frac{\pi}{2}$. To the author's knowledge, there are no results for these cases. One purpose of this paper is to establish results on the existence of Kaplan-Yorke periodic solutions with period 4 for these situations.

To study the existence of Kaplan-Yorke periodic solutions of (1.1) with period 4, we rely on Hamiltonian systems. Let $x$ be a Kaplan-Yorke periodic solution of (1.1) with period 4. Set $y(t)=x(t-1)$. Then $(x, y)$ satisfies the following system of ordinary differential equations:

$$
\left\{\begin{array}{l}
x^{\prime}(t)=-f(y(t)) \\
y^{\prime}(t)=f(x(t))
\end{array}\right.
$$

Define $F(x)=\int_{0}^{x} f(z) d z$. Then $F(x)$ is strictly increasing for $x \geq 0$ since $f$ satisfies (1.2). Obviously, $H(x, y)=F(x)+F(y)$ is the Hamiltonian of (1.5). On every level set of the Hamiltonian of (1.5) there is exactly one orbit of the system. For $c>0$, the solution $\left(x_{c}(t), y_{c}(t)\right)$ of 1.5 with the initial condition $(x(0), y(0))=(c, 0)$ is periodic and its minimal period is denoted by $T(c)$. As $F\left(x_{c}(t)\right)+F\left(y_{c}(t)\right)=F(c)$, it follows that the closed orbit $\Gamma_{c}=\left\{\left(x_{c}(t), y_{c}(t)\right) \mid t \in\right.$ $\mathbb{R}\}$ is symmetric with respect to the $x$-axis, the $y$-axis, the lines $x=y$ and $y=-x$, respectively. We can easily prove that the four intersection points of $\Gamma_{c}$ with the two axes in the counterclockwise direction are respectively: $A=\left(x_{c}(0), y_{c}(0)\right)=$ $(c, 0), B=\left(x_{c}\left(\frac{T(c)}{4}\right), y_{c}\left(\frac{T(c)}{4}\right)\right)=(0, c), C=\left(x_{c}\left(\frac{T(c)}{2}\right), y_{c}\left(\frac{T(c)}{2}\right)\right)=(-c, 0)$ and $D=$ $\left(x_{c}\left(\frac{3 T(c)}{4}\right), y_{c}\left(\frac{3 T(c)}{4}\right)\right)=(0,-c)$. Now let $E$ be the first intersection point of $\Gamma_{c}$ with the line $y=x$. Then $E=\left(x_{c}\left(\frac{T(c)}{8}\right), x_{c}\left(\frac{T(c)}{8}\right)\right)=\left(F^{-1}\left(\frac{1}{2} F(c)\right), F^{-1}\left(\frac{1}{2} F(c)\right)\right)$. 
Let $\theta_{c}(t)$ be the angle from the positive $x$-axis to the ray from $(0,0)$ to $\left(x_{c}(t), y_{c}(t)\right)$. Then $\tan \left(\theta_{c}(t)\right)=\frac{y_{c}(t)}{x_{c}(t)}$. This quantity will play an important role in our discussion. It follows from (1.5) that

$$
\theta_{c}^{\prime}(t)=\frac{x_{c}(t) f\left(x_{c}(t)\right)+y_{c}(t) f\left(y_{c}(t)\right)}{x_{c}^{2}(t)+y_{c}^{2}(t)} .
$$

Now, we are ready to present the main results of this paper.

\section{MAIn RESUlts AND PROOFs}

The Kaplan-Yorke Theorem tells us that (1.1) has a Kaplan-Yorke periodic solution with period 4 when $\min \{\alpha, \beta\}<\frac{\pi}{2}<\max \{\alpha, \beta\}$. In this section, we will see what will happen when this condition is not satisfied. First, we present a result on the nonexistence of a Kaplan-Yorke periodic solution.

Theorem 2.1. Assume that either $\frac{f(x)}{x} \geq \frac{\pi}{2}$ for $x \neq 0$ or $\frac{f(x)}{x} \leq \frac{\pi}{2}$ for $x \neq 0$. If $\frac{f(x)}{x}$ is not the constant function $\frac{\pi}{2}$ in any neighborhood of 0 , then (1.1) has no Kaplan-Yorke periodic solution with period 4.

Proof. We first consider the case where $\frac{f(x)}{x} \geq \frac{\pi}{2}$ for $x \neq 0$. Given $c>0$, for $0 \leq t \leq T(c)$, it follows from (1.6) that

$$
\theta_{c}^{\prime}(t) \geq \frac{\frac{\pi}{2} x_{c}^{2}(t)+\frac{\pi}{2} y_{c}^{2}(t)}{x_{c}^{2}(t)+y_{c}^{2}(t)}=\frac{\pi}{2} .
$$

Moreover, the inequality (2.1) is strict at some $t \in[0, T(c)]$ as $\frac{f(x)}{x}$ is not the constant function $\frac{\pi}{2}$ in any neighborhood of 0 . Then integrating (2.1) on the interval $[0, T(c)]$ yields

$$
2 \pi=\int_{0}^{T(c)} \theta_{c}^{\prime}(t) d t>\frac{\pi}{2} T(c),
$$

which implies that $T(c)<4$. The case $\frac{f(x)}{x} \leq \frac{\pi}{2}$ for $x \neq 0$ will similarly lead to $T(c)>4$. Thus, in either case, (1.1) has no Kaplan-Yorke periodic solution with period 4 and this completes the proof.

Example 2.2. Consider the delay differential equation

$$
x^{\prime}(t)=-\eta\left[1-x^{2}(t)\right] x(t-1),
$$

where $\eta>0$. We concentrate on solutions with values in the interval $(-1,1)$. Let $y(t)=\frac{1}{2} \ln \frac{1+x(t)}{1-x(t)}$. Then $y$ satisfies

$$
y^{\prime}(t)=-f(y(t-1)),
$$

where $f(v)=\eta\left(e^{2 v}-1\right) /\left(1+e^{2 v}\right)$. Obviously, $f$ is odd and $v f(v)>0$ for $v \neq 0$. It is easy to see that $\lim _{v \rightarrow 0} \frac{f(v)}{v}=\eta$ and $\lim _{v \rightarrow \infty} \frac{f(v)}{v}=0$. It follows from the Kaplan-Yorke Theorem that (2.2) has a Kaplan-Yorke periodic solution with period 4 when $\eta>\frac{\pi}{2}$. With the help of Theorem [2.1, we prove that the condition $\eta>\frac{\pi}{2}$ is also necessary for (2.2) to have a Kaplan-Yorke periodic solution with period 4 . It is clear that $\frac{f(v)}{v}$ is not a constant function in any neighborhood of 0 . Now, we show that $\frac{e^{2 v}-1}{1+e^{2 v}}<v$ for $v \neq 0$ or equivalently

$$
g(v):=(1-v) e^{2 v}-1-v<0 \quad \text { for } v>0 .
$$


Clearly, the inequality (2.3) is true when $v \geq 1$. When $0<v<1$, by a simple calculation, we get

$$
g(0)=0 \quad \text { and } \quad g^{\prime}(v)=(1-2 v) e^{2 v}-1
$$

and

$$
g^{\prime}(0)=0 \quad \text { and } \quad g^{\prime \prime}(v)=-4 v e^{2 v} .
$$

It follows from $(2.5)$ that $g^{\prime}(v)<0$ for $v>0$. This, combined with (2.4), immediately yields (2.3). Therefore, $\frac{f(v)}{v} \leq \frac{\pi}{2}$ for $v \neq 0$ when $\eta \leq \frac{\pi}{2}$. By Theorem 2.1, equation (2.2) has no Kaplan-York periodic solution with period 4. Hence (2.2) has a Kaplan-Yorke periodic solution with period 4 if and only if $\eta>\frac{\pi}{2}$.

The assumptions in Theorem 2.1 mean that for $x>0$, the graph of the function $f(x)$ remains either above or below the line $y=\frac{\pi}{2} x$ and does not agree with $y=\frac{\pi}{2} x$ on the segment containing the origin. Therefore, if (1.1) has a KaplanYorke periodic solution with period 4, then the graph of $f(x)$ must cross the line $y=\frac{\pi}{2} x$, transversally, at least once at some $x>0$. By symmetry, a similar conclusion also holds on the $x<0$ side.

Theorem 2.3. Assume that $\alpha<\frac{\pi}{2}$ and

$$
\lim _{x \rightarrow \infty} x^{2}\left(\frac{f(x)}{x}-\frac{\pi}{2}\right)=\infty .
$$

Then (1.1) has at least one Kaplan-Yorke periodic solution with period 4.

Proof. It follows from (2.6) that $\frac{f(x)}{x}>\frac{\pi}{2}$ for all $x$ large enough. This, combined with the fact that $\alpha<\frac{\pi}{2}$, implies that there exists $h>0$ such that $f(h)=\frac{\pi h}{2}$ and

$$
f(x)>\frac{\pi}{2} x \quad \text { for } x \geq h .
$$

It follows from (2.7) that $F(x) \rightarrow \infty$ as $x \rightarrow \infty$. For $c>F^{-1}(2 F(h))$, let $t_{c} \in$ $\left(0, \frac{T(c)}{4}\right)$ such that $y_{c}\left(t_{c}\right)=h$. Then $t_{c} \in\left(0, \frac{T(c)}{8}\right)$ as $y_{c}\left(\frac{T(c)}{8}\right)=F^{-1}\left(\frac{F(c)}{2}\right)>h$. According to Kaplan and Yorke [9], we know that $T\left(0^{+}\right)=\frac{2 \pi}{\alpha}$ and hence $T\left(0^{+}\right)>4$ since $\alpha<\frac{\pi}{2}$. In order to complete the proof, it suffices to show that there exists $c_{0}>0$ such that $T\left(c_{0}\right)<4$ since $T(c)$ is a continuous function of $c$ for $c \in(0, \infty)$. By way of contradiction, we assume that $T(c) \geq 4$ for all $c>0$. In particular, for any $c>F^{-1}(2 F(h))$, denote $\overline{\theta_{c}}(t)=\theta_{c}(t)-\frac{\pi}{2} t$. Then $\overline{\theta_{c}}(0)=0,{\overline{\theta_{c}}}^{\prime}(0)=\frac{f(c)}{c}-\frac{\pi}{2}>0$ and $\overline{\theta_{c}}\left(\frac{T(c)}{8}\right)=\frac{\pi}{4}-\frac{\pi}{2} \cdot \frac{T(c)}{8} \leq 0$. It follows that there exists $\xi_{c} \in\left(0, \frac{T(c)}{8}\right)$ such that ${\overline{\theta_{c}}}^{\prime}\left(\xi_{c}\right)=0$, i.e., $\theta_{c}^{\prime}\left(\xi_{c}\right)=\frac{\pi}{2}$. With the help of (1.6) and (2.7), we note that $\theta_{c}^{\prime}(t)>\frac{\pi}{2}$ for $t_{c} \leq t \leq \frac{T(c)}{8}$. This gives $\xi_{c} \in\left(0, t_{c}\right)$. Then $0<y_{c}\left(\xi_{c}\right)<y_{c}\left(t_{c}\right)=h$ and hence $x_{c}\left(\xi_{c}\right) \rightarrow \infty$ as $c \rightarrow \infty$ by $F(c)=F\left(x_{c}\left(\xi_{c}\right)\right)+F\left(y_{c}\left(\xi_{c}\right)\right)<F\left(x_{c}\left(\xi_{c}\right)\right)+F(h)$ and $F(c) \rightarrow \infty$ as $c \rightarrow \infty$. Moreover, there exist a subsequence $\left\{y_{c_{n}}\left(\xi_{c_{n}}\right)\right\}$ of $\left\{y_{c}\left(\xi_{c}\right)\right\}$ and $p \in[0, h]$ such that $y_{c_{n}}\left(\xi_{c_{n}}\right) \rightarrow p$ as $n \rightarrow \infty$. It follows from $\theta_{c_{n}}^{\prime}\left(\xi_{c_{n}}\right)=\frac{\pi}{2}$ that

$$
x_{c_{n}}^{2}\left(\xi_{c_{n}}\right)\left(\frac{f\left(x_{c_{n}}\left(\xi_{c_{n}}\right)\right)}{x_{c_{n}}\left(\xi_{c_{n}}\right)}-\frac{\pi}{2}\right)=y_{c_{n}}^{2}\left(\xi_{c_{n}}\right)\left(\frac{\pi}{2}-\frac{f\left(y_{c_{n}}\left(\xi_{c_{n}}\right)\right)}{y_{c_{n}}\left(\xi_{c_{n}}\right)}\right) .
$$


Letting $n \rightarrow \infty$ gives us

$$
\begin{aligned}
\lim _{x \rightarrow \infty} x^{2}\left(\frac{f(x)}{x}-\frac{\pi}{2}\right) & =\lim _{n \rightarrow \infty} x_{c_{n}}^{2}\left(\xi_{c_{n}}\right)\left(\frac{f\left(x_{c_{n}}\left(\xi_{c_{n}}\right)\right)}{x_{c_{n}}\left(\xi_{c_{n}}\right)}-\frac{\pi}{2}\right) \\
& =\lim _{n \rightarrow \infty} y_{c_{n}}^{2}\left(\xi_{c_{n}}\right)\left(\frac{\pi}{2}-\frac{f\left(y_{c_{n}}\left(\xi_{c_{n}}\right)\right)}{y_{c_{n}}\left(\xi_{c_{n}}\right)}\right) \\
& =p^{2}\left(\frac{\pi}{2}-\frac{f(p)}{p}\right) \\
& <\infty,
\end{aligned}
$$

a contradiction to (2.6). This completes the proof.

Remark 2.4. Note that (2.6) automatically holds when $\beta>\frac{\pi}{2}$. Therefore, Theorem 2.3 greatly improves the Kaplan-Yorke Theorem.

Example 2.5. Let $f(x)=\frac{\pi}{2} x\left(1.2-0.2 \cos x+\left(x^{2}-\frac{1}{4}\right) /\left(1+x^{8 / 3}\right)\right)$. For this function, $\alpha=\frac{3 \pi}{8}<\frac{\pi}{2}$ but $\beta$ does not exist. Thus the Kaplan-Yorke Theorem is inapplicable. However, the assumptions of Theorem 2.3 are satisfied and hence (1.1) has at least one Kaplan-Yorke periodic solution with period 4.

Theorem 2.6. Assume that there exist positive constants $b$ and $d$ with $b<$ $F^{-1}\left(\frac{1}{2} F(d)\right)$ such that

$$
f(x) \leq \frac{\pi}{2} x \quad \text { for } 0 \leq x \leq b
$$

and

$$
f(x) \geq \frac{\pi}{2} x \quad \text { for } b \leq x \leq d .
$$

Moreover, suppose $\frac{f(x)}{x}$ is not the constant function $\frac{\pi}{2}$ in any neighborhood of 0 and

$$
f(x) \geq \frac{\pi}{2} x+\frac{M}{x} \quad \text { for } d_{1} \leq x \leq d,
$$

where $M=\max _{0 \leq y \leq b}\left(\frac{\pi}{2} y^{2}-y f(y)\right)$ and $d_{1}>0$ satisfies $F\left(d_{1}\right)+F(b)=F(d)$. Then (1.1) has at least one Kaplan-Yorke periodic solution with period 4.

Proof. It suffices to show that there exists a $c>0$ such that $T(c)=4$. As $T(c)$ is continuous in $c$, we can complete the proof by proving $T(d)<4$ and

$$
T(c)>4 \quad \text { for } 0<c<b .
$$

We first proceed by way of contradiction to prove that $T(d)<4$. Assume that $T(d) \geq 4$. Let $\overline{\theta_{d}}(t)=\theta_{d}(t)-\frac{\pi}{2} t$. Then by (2.10),

$$
\left\{\begin{aligned}
\overline{\theta_{d}}(0) & =0, \\
\overline{\theta_{d}}\left(\frac{T(d)}{8}\right) & =\frac{\pi}{4}-\frac{\pi}{2} \cdot \frac{T(d)}{8} \leq 0, \\
{\overline{\theta_{d}}}^{\prime}(0) & =\frac{f(d)}{d}-\frac{\pi}{2}>0 .
\end{aligned}\right.
$$

Let $\xi \in\left(0, \frac{T(d)}{8}\right)$ be the smallest such that ${\overline{\theta_{d}}}^{\prime}(\xi)=0$ or $\theta_{d}^{\prime}(\xi)=\frac{\pi}{2}$. Now let $\bar{t} \in\left(0, \frac{T(d)}{4}\right)$ such that $y_{d}(\bar{t})=b$. Then $\bar{t}<\frac{T(d)}{8}$ as $y_{d}\left(\frac{T(d)}{8}\right)=F^{-1}\left(\frac{1}{2} F(d)\right)>b$. It follows that $x_{d}(\bar{t})=d_{1}$ and hence by (2.9) and (2.10),

$$
\theta_{d}^{\prime}(t)>\frac{\pi}{2} \quad \text { for } \bar{t}<t \leq \frac{T(d)}{8} .
$$

This implies that $\xi<\bar{t}$. So

$$
d_{1}=x_{d}(\bar{t})<x_{d}(\xi)<d \quad \text { and } \quad 0<y_{d}(\xi)<y_{d}(\bar{t})=b .
$$


As $\theta_{d}^{\prime}(t)>\frac{\pi}{2}$ for $t \in[0, \xi)$, there exists $\eta \in(\xi, \bar{t})$ such that $\theta_{d}^{\prime}(\eta)<\frac{\pi}{2}$. Otherwise, $\theta_{d}^{\prime}(t) \geq \frac{\pi}{2}$ for $t \in\left[0, \frac{T(d)}{8}\right]$. This gives $\frac{\pi}{4}>\frac{\pi}{2} \cdot \frac{T(d)}{8}$ and hence $T(d)<4$, a contradiction to the assumption that $T(d) \geq 4$. Obviously,

$$
d_{1}=x_{d}(\bar{t})<x_{d}(\eta)<x_{d}(\xi)<d \quad \text { and } \quad 0<y_{d}(\xi)<y_{d}(\eta)<y_{d}(\bar{t})=b .
$$

In view of (2.10), we have

or

$$
f\left(x_{d}(\eta)\right) \geq \frac{\pi}{2} x_{d}(\eta)+\frac{M}{x_{d}(\eta)}
$$

$$
x_{d}(\eta) f\left(x_{d}(\eta)\right)-\frac{\pi}{2} x_{d}^{2}(\eta) \geq M \geq \frac{\pi}{2} y_{d}^{2}(\eta)-y_{d}(\eta) f\left(y_{d}(\eta)\right) .
$$

This gives $\theta_{d}^{\prime}(\eta) \geq \frac{\pi}{2}$, a contradiction to the choice of $\eta$. This proves that $T(d)<4$.

Now, we prove (2.11). In fact, for $0<c<b$, we have from (1.6) and (2.8) that

$$
\theta_{c}^{\prime}(t)=\frac{x_{c}(t) f\left(x_{c}(t)\right)+y_{c}(t) f\left(y_{c}(t)\right)}{x_{c}^{2}(t)+y_{c}^{2}(t)} \leq \frac{\pi}{2} .
$$

As before, the equality in the above inequality (2.12) does not always hold since $\frac{f(x)}{x}$ is not the constant function $\frac{\pi}{2}$ in any neighborhood of 0. Integrating (2.12) over the interval $[0, T(c)]$, we get $2 \pi<\frac{\pi}{2} T(c)$. This immediately gives (2.11) and the proof is complete.

In Theorem 2.3 and Theorem [2.6 we assumed that $\alpha \leq \frac{\pi}{2}$. When $\alpha \geq \frac{\pi}{2}$, we can have the following two results, the proofs of which are similar to those of Theorem 2.3 and Theorem 2.6 and thus are omitted.

Theorem 2.7. Assume that $\alpha>\frac{\pi}{2}, \lim _{x \rightarrow \infty} x^{2}\left(\frac{\pi}{2}-\frac{f(x)}{x}\right)=\infty$ and $\lim _{x \rightarrow \infty} F(x)=$ $\infty$. Then (1.1) has at least one Kaplan-Yorke periodic solution with period 4.

Theorem 2.8. Assume that there exist positive constants $b$ and $d$ with $b<$ $F^{-1}\left(\frac{1}{2} F(d)\right)$ such that

$$
f(x) \geq \frac{\pi}{2} x \text { for } 0 \leq x \leq b \quad \text { and } \quad f(x) \leq \frac{\pi}{2} x \text { for } b \leq x \leq d .
$$

Moreover, suppose $\frac{f(x)}{x}$ is not the constant function $\frac{\pi}{2}$ in any neighborhood of 0 and

$$
f(x) \leq \frac{\pi}{2} x-\frac{m}{x} \text { for } d_{1} \leq x \leq d,
$$

where $m=\max _{0 \leq y \leq b}\left(y f(y)-\frac{\pi}{2} y^{2}\right)$ and $d_{1}>0$ satisfies $F\left(d_{1}\right)+F(b)=F(d)$. Then (1.1) has at least one Kaplan-Yorke periodic solution with period 4.

In Theorems 2.3. 2.6 2.8, we obtained some new sufficient conditions on the existence of at least one Kaplan-Yorke periodic solution with period 4 . In the following, we present some results on the existence of at least two such periodic solutions.

Theorem 2.9. Under the assumptions of Theorem 2.6, if $\max \{\alpha, \beta\}<\frac{\pi}{2}$, then (1.1) has at least two Kaplan-Yorke periodic solutions with period 4.

Proof. According to Kaplan and Yorke [9], we have $T\left(0^{+}\right)=\frac{2 \pi}{\alpha}>4$ and $T(\infty)=$ $\frac{2 \pi}{\beta}>4$. From the proof of Theorem 2.6 , we know that $T(d)<4$. Then it is easy to see that there exist $c_{1}$ and $c_{2}$ such that $0<c_{1}<c_{2}$ and $T\left(c_{1}\right)=T\left(c_{2}\right)=4$. This completes the proof.

Similarly, from the proofs of Theorem [2.6. Theorem 2.8 and Theorem 2.7, we can obtain the following results. 
Theorem 2.10. Under the assumptions of Theorem 2.8 , if $\min \{\alpha, \beta\}>\frac{\pi}{2}$, then (1.1) has at least two Kaplan-Yorke periodic solutions with period 4.

Theorem 2.11. Under the assumptions of Theorem 2.6, if $\alpha<\frac{\pi}{2}$ and $\lim _{x \rightarrow \infty} x^{2}\left(\frac{\pi}{2}-\right.$ $\left.\frac{f(x)}{x}\right)=\infty$, then (1.1) has at least two Kaplan-Yorke periodic solutions with period 4 .

Example 2.12. Let

where

$$
f(x)= \begin{cases}f_{0}(x), & x \in[0, \infty) \\ -f_{0}(-x), & x<0\end{cases}
$$

$$
f_{0}(x)= \begin{cases}x\left(x-1+\frac{\pi}{2}\right), & 0 \leq x \leq 2, \\ x\left(-\frac{3}{4} x+\frac{\pi+5}{2}\right), & 2 \leq x \leq 4, \\ \frac{\pi-1}{2} x, & x \geq 4\end{cases}
$$

Obviously, $f \in C(\mathbb{R}, \mathbb{R}), f$ is odd, $x f(x)>0$ for $x \neq 0$ and $\alpha=\beta=\frac{\pi}{2}-\frac{1}{2}<\frac{\pi}{2}$. Then the Kaplan-Yorke Theorem cannot be applied. By a simple computation, we have

where

$$
F(x)= \begin{cases}F_{0}(x), & x \geq 0 \\ F_{0}(-x), & x<0\end{cases}
$$

$$
F_{0}(x)= \begin{cases}\frac{1}{3} x^{3}+\frac{1}{2}\left(\frac{\pi}{2}-1\right) x^{2}, & 0 \leq x \leq 2, \\ -\frac{1}{4} x^{3}+\frac{\pi+5}{4} x^{2}-\frac{7}{3}, & 2 \leq x \leq 4, \\ \frac{\pi-1}{4} x^{2}+\frac{17}{3}, & x \geq 4\end{cases}
$$

Choose $b=1$ and $d=2$. Then $f(1)=\frac{\pi}{2}, f(x)<\frac{\pi}{2} x$ for $0<x<1$ and $f(x)>\frac{\pi}{2} x$ for $1<x<2$. Note that $F(1)=\frac{1}{3}+\frac{\pi}{4}-\frac{1}{2}=\frac{\pi}{4}-\frac{1}{6}, F(2)=\pi+\frac{2}{3}, F\left(d_{1}\right)=\frac{\pi}{2}+\frac{1}{3}$. Since $F(x)$ is strictly increasing in $[0,2]$ and $F(\sqrt{2})=\frac{2 \sqrt{2}}{3}+\frac{\pi}{2}-1<\frac{\pi}{2}+\frac{1}{3}=F\left(d_{1}\right)$, we have $\sqrt{2}<d_{1}$. For $\sqrt{2} \leq x \leq 2$,

$$
f(x)=-\frac{\pi}{2} x+x(x-1) \geq \frac{\pi}{2} x+\frac{2(\sqrt{2}-1)}{x}>\frac{\pi}{2} x+\frac{M}{x},
$$

where $M=\max _{0 \leq y \leq 1}\left[\frac{\pi}{2} y^{2}-y f(y)\right]=\max _{0 \leq y \leq 1} y^{2}(1-y)=\frac{4}{27}$. So we have verified all the assumptions of Theorem 2.6. Therefore, by Theorem 2.9] we know that (1.1) has at least two Kaplan-Yorke periodic solutions with period 4.

Finally, we discuss the case where $\alpha=\beta=\frac{\pi}{2}$.

Theorem 2.13. Assume that $\alpha=\beta=\frac{\pi}{2}$ and that there exists $b>0$ such that $\frac{f(x)}{x} \leq \frac{\pi}{2}$ for $0 \leq x \leq b$ and $\frac{f(x)}{x}$ is not the constant function $\frac{\pi}{2}$ in any neighborhood of 0 . If $\lim _{x \rightarrow \infty} x^{2}\left(\frac{f(x)}{x}-\frac{\pi}{2}\right)=\infty$, then (1.1) has at least one Kaplan-Yorke periodic solution with period 4 .

Proof. It follows from the proofs of Theorem 2.1 and Theorem 2.3 that $T(c)>4$ for $0<c<b$ and $T\left(c_{0}\right)<4$ for some $c_{0}>0$, respectively. Therefore, there exists $c^{*}>0$ such that $T\left(c^{*}\right)=4$. This completes the proof.

Example 2.14. Let

$$
f(x)=\frac{\pi}{2} x\left(1+\frac{x^{2}\left(x^{2}-1\right)}{1+x^{14 / 3}}\right) .
$$

It is easy to check that the assumptions of Theorem 2.13 are satisfied and hence (1.1) has at least one Kaplan-Yorke periodic solution with period 4. 
We can also obtain the following result from the proofs of Theorem 2.3 and Theorem 2.8.

Theorem 2.15. Under the assumptions of Theorem 2.8, if $\alpha=\beta=\frac{\pi}{2}$ and $\lim _{x \rightarrow \infty} x^{2}\left(\frac{f(x)}{x}-\frac{\pi}{2}\right)=\infty$, then (1.1) has at least two Kaplan-Yorke periodic solutions with period 4 .

\section{REFERENCES}

1. Y. S. Chen, The existence of periodic solutions of the equation $x^{\prime}(t)=-f(x(t), x(t-\tau))$, J. Math. Anal. Appl., 163 (1992) 227-237. MR1144718 (92m:34152)

2. G. Fei, Multiple periodic solutions of differential delay equations via Hamiltonian systems. I. Nonlinear Anal., 65 (2006) 25-39. MR2226257 (2007f:34118)

3. G. Fei, Multiple periodic solutions of differential delay equations via Hamiltonian systems. II. Nonlinear Anal., 65 (2006) 40-58. MR2226258(2007f:34119)

4. W. G. Ge, The number of simple periodic solutions to the differential-difference equation $\dot{x}(t)=f(x(t-1))$, Chinese Ann. Math. Ser. A, 14 (1993) 472-479. MR1243330 (94k:34130)

5. Z. Guo, Existence and estimate of the number of nontrivial periodic solutions for delay differential equations, Appl. Math. J. Chinese Univ., 25 (2010), 134-140. MR2767084

6. Z. Guo and J. Yu, Multiplicity results for periodic solutions to delay differential equations via critical point theory, J. Differential Equations, 218 (2005) 15-35. MR2174964 (2006g:34156)

7. A. V. M. Herz, Solutions of $\dot{x}(t)=-g(x(t-1))$ approach the Kaplan-Yorke orbits for odd sigmoid $g$, J. Differential Equations, 118 (1995), 36-53. MR.1329402 (96h:34140)

8. S. Jekel and C. Johnston, A Hamiltonian with periodic orbits having several delays, J. Differential Equations, 222 (2006) 425-438. MR2208051 (2007b:34162)

9. J. L. Kaplan and J. A. Yorke, Ordinary differential equations which yield periodic solutions of differential delay equations, J. Math. Anal. Appl., 48 (1974) 317-324. MR0364815 (51:1069)

10. T. Krisztin, Global dynamics of delay differential equations, Period. Math. Hungar., 56 (2008), 83-95. MR2385485(2008m:37002)

11. B. Lani-Wayda, Change of the attractor structure for $\dot{x}(t)=f(x(t-1))$ when $f$ changes from monotone to non-monotone negative feedback, J. Differential Equations, 248 (2010) 1120-1142. MR2592883 (2011d:34105)

12. J. Li and X. He, Proof and generalization of Kaplan-Yorke's conjecture under the condition $f^{\prime}(0)>0$ on periodic solution of differential delay equations, Sci. China Ser. A, 42 (1999) 957-964. MR1736586 (2000k:34113)

13. J. Li, X.-Z. He and Z. Liu, Hamiltonian symmetric groups and multiple periodic solutions of differential delay equations, Nonlinear Anal. Ser. A: Theory Methods, 35 (1999) 457-474. MR 1656528 (99j:34102)

14. J. Li, Z. Liu and X. He, Periodic solutions of some differential delay equations created by Hamiltonian systems, Bull. Austral. Math. Soc., 60 (1999) 377-390. MR1727474(2000i:34140)

15. R. D. Nussbaum, Periodic solutions of special differential equations: an example in nonlinear functional analysis, Proc. Roy. Soc. Edinburgh Sect. A, 81 (1978) 131-151. MR.529382 (80h:34097)

16. L. Wen, Existence of periodic solutions to a class of differential difference equations, Chinese Ann. Math., 10A (1989) 249-254. MR1024909 (90j:34104)

College of Mathematics and Information Sciences, Guangzhou University, Guangzhou 510006, People's Republic of China

E-mail address: jsyu@gzhu.edu.cn 